\title{
Selection of host differentials for the identification of Albugo candida pathotypes
}

\author{
PUJA PANDEY* and A.K. TEWARI \\ Department of Plant Pathology, College of Agriculture, G.B. Pant University of Agriculture and Technology, U.S. Nagar, Pantnagar \\ 263 145, Uttarakhand, India
}

Received: 11 April 2017/ Accepted: 9 June 2017/ Published online: 24 July 2017

(C) Indian Phytopathological Society 2017

\begin{abstract}
Albugo candida (A. cruciferum) the cause of the white rust/white blister of the rapeseed and mustard occurs in all cruciferous growing countries. The present investigation was conducted to study disease reaction on different $B r a s s i c a$ genotypes viz., EC-399301, NRCDR-513, EC-399296, Rohini, EC-399299, NRCDR-515, BIOYSR, EC-399313, JM-1 (B. juncea), EC-414293 (B. rapa), GSL-1 (B. napus), PBC -9221 (B. carinata) and B. nigra local during year 2010-11 and Rohini, EC399299, EC-414293, B. nigra local, EC-399296, EC-399313, EC-399301, JM-1, PBC-9221, NRCDR-515, GSL-1 using different A. candida isolates (Pantnagar, Ludhiana, Hisar, Bharatpur and Delhi) for the selection of host differentials and identification of $A$. candida pathotypes. Based on phenotypic disease reactions at cotyledonary and true leaf stage, the Brassica genotypes were grouped into a set of five host differentials. Among various disease management practices, breeding for resistant genotype/varieties is one of the most ecofriendly, economic and effective method for the management of plant diseases.
\end{abstract}

Keywords: Albugo candida pathotypes, Brassica genotypes, host differentials, white rust

The oilseed Brassica species are the most important rabi oilseed crop. Among various diseases of rapeseedmustard white rust caused by Albugo candida is one of the major constraints. It is estimated that the floral infection can cause losses from 23-54.5\% (Saharan et al., 1984) and 17-34 per cent (Vignesh et al., 2011). The information to be gathered on the occurrence of pathotypes and resistance will also be helpful in identification of new genes for resistance effective against existing virulent pathogen. The resistance of any variety may breakdown due to the attack of new virulent pathotypes of the pathogen. Therefore, use of different pathotypes/races of the pathogen is essential during screening of resistant germplasm. The different races/ pathotypes are identified by using host differentials. Four distinct and new pathotypes of Albugo candida AC-14 (RL 1359), AC-15 and AC-16 (Kranti) and AC-17 (RH 30) were identified using 11 host differentials (EC129126-1, EC-322090, EC-322092, EC-322093, Varuna, EC-287711, ZEM-1, RC 781, RH-30, RH-8113 and Rajat). AC-15 had a wider host range, while $\mathrm{AC}-17$ had the least among host differentials tested. Although some workers have developed host differentials for the identification of $A$. candida pathotypes, still lacking a known set of host differentials (Gupta and Saharan, 2002).

White rust infected leaves from different locations of India viz., Pantnagar, Ludhiana, Hisar, Bharatpur and Delhi during 2010-11 and 2011-12 were collected. Single

\footnotetext{
${ }^{\star}$ Corresponding author: pujapandey41124@gmail.com
}

rust pustule of each isolate along with a small portion of the leaf was collected and stored at refrigerator temperature $\left(4^{\circ} \mathrm{C}\right)$ in glass vials for further studies. Sporangia were obtained from single white rust pustule of each isolate with the help of a needle. Sporangial suspension of each isolate was made in a test tube containing sterilized distilled water. The suspension was kept at $18^{\circ} \mathrm{C}$ for $4 \mathrm{hrs}$ for germination of sporangia i.e. release of zoospores. The inoculum of each isolate was purified and multiplied separately on seedlings of susceptible mustard cultivar (Varuna) grown in plastic pots $(15 \mathrm{~cm}$ in diam.) under glasshouse conditions.

The zoospores suspension $(2.5 \times 105 / \mathrm{ml})$ of each isolate was adjusted with the help of haemocytometer and sprayed at cotyledonary stage i.e. 7 days after sowing (DAS) using atomizer. The inoculated plants were transferred in plant propagator for $72 \mathrm{hrs}$ to provide 80$90 \% \mathrm{RH}$ and kept in glasshouse at $18 \pm 2^{\circ} \mathrm{C}$ for infection. After 72 hrs pots were taken out from the propagator. The pure single rust pustules of each isolate was collected 12 DAS and used for the preparation of zoospore suspension as described earlier. The zoospore suspension of each isolate was sprayed on each Brassica cultivar viz., EC-399301, NRCDR-513, EC-399296, Rohini, EC-399299, NRCDR-515, BIOYSR, EC-399313, JM-1 (B. juncea), EC-414293 (B. rapa), GSL-1 (B. napus), PBC-9221 (B. carinata) and B. nigra local at cotyledonary stage i.e. 7 in two consequent years i.e. 2010-11 and 2011-12 and kept in plant propagator for $72 \mathrm{hrs}$ and glasshouse as described earlier. Optimum soil moisture 
Table 1. Phenotypic disease reaction

\begin{tabular}{lllc}
\hline Interaction phenotype & Host response & Pathogen growth & Disease score \\
\hline NN & No response & No sporulation & 0 \\
F(N) & Light necrotic flecking & No sporulation & 1 \\
FN & Heavy necrotic flecking & No sporulation & 2 \\
S1 & Any host response & Minute pustule on upper leaf surface (mp) & 3 \\
S2 & Any host response & i) Few pustules (FP) on lower leaf surface & 4 \\
& & ii) Numerous pustules (NP) on lower leaf surface & 5 \\
S3 & Any host response & i) Large scattered (LP) (CP) on lower leaf surface & 6 \\
& & ii) Coalescing pustules (CP) on lower leaf surface & 7 \\
\hline
\end{tabular}

Table 2. Grouping of host differentials based on phenotypic disease reactions at cotyledonary stage

\begin{tabular}{|c|c|c|c|c|c|}
\hline \multirow[t]{2}{*}{ Genotype } & \multicolumn{5}{|c|}{ Albugo candida isolate } \\
\hline & Bharatpur & Delhi & Hisar & Ludhiana & Pantnaga \\
\hline \multicolumn{6}{|l|}{ Group I } \\
\hline EC-399301 & S3-6 & S3-7 & S3-7 & S3-7 & S3-7 \\
\hline Ec-399296 & S3-6 & S3-6 & S3-6 & S3-6 & S3-6 \\
\hline EC-399299 & S3-7 & S3-7 & S3-6 & S3-6 & S3-7 \\
\hline EC-399313 & S3-6 & S3-6 & S3-6 & S3-7 & S3-6 \\
\hline B. nigra local & S3-6 & S3-7 & S3-7 & S3-7 & S3-7 \\
\hline Rohini & S3-7 & S3-6 & S3-6 & S3-6 & S3-7 \\
\hline \multicolumn{6}{|l|}{ Group II } \\
\hline $\mathrm{JM}-1$ & S2-4 & S2-5 & S2-5 & S2-4 & S2-5 \\
\hline \multicolumn{6}{|l|}{ Group III } \\
\hline EC-414293 & $\mathrm{NN}-0$ & $\mathrm{NN}-\mathrm{O}$ & S3-7 & $\mathrm{NN}-0$ & S3-6 \\
\hline \multicolumn{6}{|l|}{ Group IV } \\
\hline NRCDR-513 & $\mathrm{NN}-0$ & $\mathrm{NN}-0$ & $\mathrm{NN}-0$ & S3-6 & $\mathrm{NN}-0$ \\
\hline BIOYSR & $\mathrm{NN}-0$ & S3-6 & NN-O & $\mathrm{NN}-0$ & $\mathrm{NN}-\mathrm{O}$ \\
\hline \multicolumn{6}{|l|}{ Group V } \\
\hline NRCDR-515 & $\mathrm{NN}-0$ & $\mathrm{NN}-\mathrm{O}$ & $\mathrm{NN}-0$ & $\mathrm{NN}-0$ & $\mathrm{NN}-0$ \\
\hline GSL-1 & $\mathrm{NN}-0$ & $\mathrm{NN}-\mathrm{O}$ & $\mathrm{NN}-\mathrm{O}$ & $\mathrm{NN}-\mathrm{O}$ & $\mathrm{NN}-\mathrm{O}$ \\
\hline PBC-9221 & $\mathrm{NN}-0$ & $\mathrm{NN}-0$ & $\mathrm{NN}-0$ & NN-O & $\mathrm{NN}-0$ \\
\hline
\end{tabular}

$(80-85 \%)$ and temperature $\left(18-20^{\circ} \mathrm{C}\right)$ was maintained in glasshouse for the development of symptoms. Phenotypic disease reaction in each genotype against each isolate was recorded at cotyledonary stage (13 DAI) using 0-7 rating scale.

Based on the phenotypic disease reaction, the Brassica genotypes tested in the present investigation were grouped into 5 different groups (Table 2). Further observations were recorded on the basis of phenotypic disease reactions for final grouping of host differentials at cotyledonary stage. The Brassica genotypes viz., EC399301, EC-399296, EC-399299, EC-399313, B. nigra local and Rohini showed disease reactions of S3-6 type (large scattered pustules) to S3-7 type (large coalescing pustules) on the lower surface against all of the isolates. Since in all these genotypes the interaction phenotype was S3 type due to this these genotypes are kept in Group I as host differentials. The genotype JM-1 showed disease reactions of S2-4 type (few pustules) and S2-5 type (numerous pustules) on lower surface against all the isolates hence kept in Group II as host differentials. EC-414293 showed disease reactions of S3-6 and S3-7 type only against two isolates i.e. Pantnagar and Hisar, while it showed immune responses against other isolates i.e. Bharatpur, Ludhiana and Delhi, hence kept in another group i.e. Group III. NRCDR 513 and BIOYSR showed S3-6 type of disease reaction only against one isolate i.e. Ludhiana and Delhi respectively, while showed immune reaction against all isolates, hence kept in Group IV and NRCDR-515, GSL-1 and PBC-9221 showed immune reaction against all isolates, hence kept in Group V.

At true leaf stage (Table 3) little variation in disease severity was found with respect to cotyledonary stage against different isolates. In most of the cases these genotypes showed S2-4 type disease reactions, while one (Rohini against Pantnagar isolate) showed S2-5 type and one (B. nigra local against Bharatpur isolate) showed S3-6 type of reaction, hence, all these genotypes were kept in Group I, JM-1 showed S2-4 to S2-5 type of disease reactions with different isolates and hence kept under Group II, EC-414293 showed S2-4 type of disease reaction with 4 of the isolates, while this genotype showed immune reactions with Delhi isolate hence kept under Group III. BIOYSR showed S2-4 type of disease reaction only with Delhi isolate, while immune reaction with other 4 isolates and hence kept in Group IV. NRCDR-513, NRCDR-515, GSL-1 and PBC-9221 showed immune type reaction to all of the isolates, hence kept under Group V. 
Table 3. Grouping of host differentials based on phenotypic disease reactions at true leaf stage

\begin{tabular}{|c|c|c|c|c|c|}
\hline \multirow[t]{2}{*}{ Genotype } & \multicolumn{5}{|c|}{ Albugo candida isolate } \\
\hline & Bharatpur & Delhi & Hisar & Ludhiana & Pantnagar \\
\hline \multicolumn{6}{|l|}{ Group I } \\
\hline EC-399301 & S2-4 & S2-4 & S2-4 & S2-4 & S2-4 \\
\hline Ec-399296 & S2-4 & S2-4 & S2-4 & S2-4 & S2-4 \\
\hline EC-399299 & S2-4 & S2-4 & S2-4 & S2-4 & S2-4 \\
\hline EC-399313 & S2-4 & S2-4 & S2-4 & S2-4 & S2-4 \\
\hline B. nigra local & S3-6 & S2-4 & S2-4 & S2-4 & S2-4 \\
\hline Rohini & S2-4 & S2-4 & S2-4 & S2-4 & S2-5 \\
\hline \multicolumn{6}{|l|}{ Group II } \\
\hline $\mathrm{JM}-1$ & S2-5 & S2-5 & S2-4 & S2-5 & S2-5 \\
\hline \multicolumn{6}{|l|}{ Group III } \\
\hline EC-414293 & S2-4 & $\mathrm{NN}-0$ & S2-4 & S2-4 & S2-4 \\
\hline \multicolumn{6}{|l|}{ Group IV } \\
\hline BIOYSR & $\mathrm{NN}-0$ & S2-4 & NN-0 & NN-0 & NN-0 \\
\hline \multicolumn{6}{|l|}{ Group V } \\
\hline NRCDR-513 & NN-0 & NN-0 & NN-0 & NN-0 & NN-0 \\
\hline NRCDR-515 & NN-0 & $\mathrm{NN}-0$ & $\mathrm{NN}-0$ & NN-0 & NN-O \\
\hline GSL-1 & NN-0 & NN-0 & NN-0 & NN-0 & NN-0 \\
\hline PBC-9221 & NN-O & $\mathrm{NN}-0$ & $\mathrm{NN}-0$ & NN-O & NN-O \\
\hline
\end{tabular}

The present study has been conducted by taking the reference of several workers. Gupta and Saharan (2002) identified four distinct and new pathotypes of Albugo candida viz. AC-14 from RL-1359, AC-15 and AC-16 from Kranti and AC-17 using 11 host differentials of $B$. juncea. Li et al. (2008) varying levels of host resistance to Australian strains of $A$. candida (race 2) were identified among the genotypes from the three countries. Kaur et al. (2011) clearly showed that the isolates obtained from $B$. juncea and $R$. raphanistrum are different in their host range. Petkowoski et al. (2010) reported that $A$. candida races of Australian isolates from B. oleracea var. italica (broccoli), B. rapa var. pekinensis (pak choi) and var. chinensis (Chinese cabbage), and Capsella bursa-pastoris (shepherd's purse) were identified on a set of Brassicaceae hosts. Bhardwaj and Sud (1993) studied reaction of Brassica cultivars against A. candida isolates from Kangra valley and reported that all cultivars of $B$. campestris were susceptible to individual and mass isolates, whereas all 13 cultivars of $B$. carinata were resistant. Of the 24 cultivars of $B$. napus, 18 showed resistant reaction to individual isolates. Of the 51 cultivars of $B$. juncea, 26 were resistant to individual and mass isolates. It is concluded that $A$. candida possess inherent variability against Brassica species. Similar work was done on same crop but with different pathogen by Akhtar et al. (2017), they detected Alternaria brassicicola in three accessions of $B$. juncea, IC-113148, Pusa Bold and Prakash. Nine isolates of $A$. candida, representing strains collected from $B$. juncea, $B$. rapa, B. oleracea, $B$. tournefortii, Raphanus raphanistrum, R. sativa, Eruca vesicaria subsp. sativa, Capsella bursa-pastoris and Sisymbrium irio, from different locations in Western Australia (W.A.), were tested on cruciferous host differentials to characterize their pathogenicity (Kaur et al., 2008).

\section{REFERENCES}

Akhtar J, Singh B, Kandan A, Chand D, Chaudhury R and Dubey SC (2017). Survival of Alternaria brassicicola in cryopreserved Brassica spp. seeds. Indian Phytopath. 70: 256257.

Bhardwaj CL and Sud AK (1993). Reaction of Brassica cultivars against Albugo candida isolates from Kangra valley. Indian Phytopath. 46: 258-260.

Gupta K and Saharan GS (2002). Identification of pathotype of Albugo Candida with stable characteristic symptoms on Indian mustard. J. Mycol. Plant Pathol. 32: 46-51.

Kaur P, Sivasithamparam K and Barbetti MJ (2008). Pathogenic behavior of strains of Albugo candida from Brassica juncea and Raphanus raphanistrum in Western Australia. Australas. Plant Pathol. 37: 353-356.

Kaur P, Sivasithamparam K and Barbetti MJ (2011). Host range and phylogenetic relationships of Albugo candida from cruciferous hosts in Western Australia, with special reference to Brassica juncea. Plant Dis. 95: 712-718.

Leckie D, Astley D, Crute I, Ellis P, Pink D, Boukema I, Monteiro AA and Dias JS (1996). The location and exploitation of genes for pest and disease resistance in European gene bank collections of horticultural brassicas. Acta Hort. 407: $95-102$.

Li CX, Sivasithamparam K, Walton G, Fels P and Barbetti MJ (2008). Both incidence and severity of white rust disease reflect host resistance in Brassica juncea germplasm from Australia, China and India. Field Crops Res. 108: 1-8.

Petkowski JE, Minchinton E, Thomson F, Faggian R and Cahill D (2010). Races of Albugo candida causing white blister rust on Brassica vegetables in Australia. Acta Hort. 867: 133-142.

Saharan GS, Kaushik CD, Gupta PP and Tripathi NN (1984). Assessment of losses and control of white rust of mustard. Indian Phytopath. 37: 397.

Vignesh M, Yadava DK, Sujata V, Yadava AK, Mohapatra T and Prabhu KV (2011). Characterization of an Indian mustard (Brassica juncea) indigenous germplasm line Bio-YSR for white rust resistance. Indian J. Plant Gen. Res. 24: 400442. 